\title{
Workflow Model Design based on Binary Tree for Process Management in PDM
}

\author{
Xuewen $\mathrm{Jin}^{1, \mathrm{a}}$, Xiaodong Zhu ${ }^{1, \mathrm{~b}}$, Yuanning $\mathrm{Liu}^{1, \mathrm{c}, \star}$, Biao Huang ${ }^{1, \mathrm{~d}}$ and Fei He $\mathrm{H}^{1, \mathrm{e}}$ \\ ${ }^{1}$ College of Software, JiLin University, Changchun 130012, China. \\ a1049406744@qq.com, bzhuxd@jlu.edu.cn, 'Tyn@jlu.edu.cn, d476738323@qq.com, ehefei10@mail \\ s.jlu.edu.cn
}

Keywords: workflow, binary tree, PDM.

\begin{abstract}
For modeling the organizational form of business activities in complex production processes, a workflow model based on binary tree for process management in PDM is presented in this paper. Take use of binary tree structure, each business activity is organized by the relationship of parent-child and brotherhood as the node of the binary tree, simulating the organization form of the actual production process. With introductions of elementary structure unit, the binary tree node, including type, state and constraints, it illustrates scheduling algorithms of node and mechanisms of control patterns and state transition. Finally, with a simple project template case study, we can find that this model is easy to implement overall control of process and has a good adaptability to process changes.
\end{abstract}

\section{Introduction}

As the key part to implement PDM project for the enterprise, process management is the core technology to realize the business process automation [2]. Take use of the theory of knowledge for detailed analysis, design and modeling not only can regulate enterprise business process, but also the established business process model is a very important knowledge base and rule base, which can guide enterprises to implement computer management information system [6]. So the application of the workflow technology is of great significance.

Modeling and supporting for dynamic process is always the problem of workflow design and implementation [1]. Traditional process modeling based on theoretical methods such as Petri nets, FSM, UML activity diagram, realizes the activity-centered and event-driven model [4-5]. With activity as the basic model unit, these models based on the graph structure, are excellent at the flexibility of transform between the activities and the convenience of the process configuration by using process control structure [3]. However, the traditional modeling methods overlooks whole process control and inherent organizational form between internal activities. Traditional model is difficult to reflect the hierarchical relationship between activities in the process of actual production and control the process as a whole.

This paper proposes a workflow model based on the binary tree. Dominated by the process, it implements a top-down and left-right process control by combining the control structure and binary tree structure. According to the basic process control structure of WFMC, it defines sequence, branch, parallel and loop patterns which are complied with its own structure. And related scheduling algorithms of activities based on this model are illustrated in the paper. This Model has advantages of clear structure and easy control.

\section{The establishment of the workflow model based on binary tree}

Basic control structure. Workflow management coalition defined 6 kinds of typical control structure for the workflow execution, they are: Sequence, AND - split, AND - join, OR-split, OR join and Loop [1-4]. In this paper, combining the control structure above with the binary tree structure, it defined the routing schema of nodes within a tree-shape workflow.

Definition. Based on the structure of binary tree, parent-child relationship and brotherhood are 
redefined in this paper.

Definition1: Right node of the node in the binary tree is defined as its brother node and this process can be iterative, namely the right node of its right node is its brother node too.

Definition2: Left node of the node in the binary tree and brother nodes of its left node are defined as its child nodes.

Workflow model based on binary tree.

Type: According to the function, nodes in the model are divided into two groups: Control node and Task node. Task node (D): Perform the specific business activity or task. Control node (C): Not perform specific tasks; on behalf of all kinds of control mode; describe the logical relations between nodes; be used to control the execution of its child nodes; can divided into: sequence(S), branch(B), parallel(P) and loop(L). Table 1 show these types of the control node.

Table 1 The control node

\begin{tabular}{|c|c|c|}
\hline Type & Pattern & Description \\
\hline $\mathrm{S}$ & squence & Child nodes should be executed in sequence. \\
\hline B & branch & $\begin{array}{c}\text { Only one child node should be executed and other child } \\
\text { nodes always suspend. }\end{array}$ \\
\hline $\mathrm{P}$ & parallel & Child nodes should be executed at the same time. \\
\hline $\mathrm{L}$ & loop & $\begin{array}{l}\text { After the implementation of all child nodes, all child } \\
\text { nodes will be re-run if meets the loop condition; Child } \\
\text { nodes should be executed in sequence. }\end{array}$ \\
\hline
\end{tabular}

Status: As an auxiliary logical expression, status can raise control ability and the adaptability of workflow model. On the one hand, it represents operation of the workflow model instance; on the other hand, state transition is used to ensure that the workflow executes the task according to the established rules automatically. Table 2 defines the status of workflow..

Table 2 The status of node

\begin{tabular}{ccc}
\hline Status & Meaning & Description \\
\hline I & Initialed & Initialization is complete. \\
J & Judging & The task is waiting for a decision. \\
E & Executing & The task is executed. \\
N & Non-executing & The task is terminated. \\
W & Waiting & The task is waiting for execution. \\
F & Finished & The task has been completed. \\
\hline
\end{tabular}

Constraint: The control node (C) must have the child node, namely its left node do exist; The task node (D) can't have the child node, namely its left node do not exist; B and P must contain two child nodes at least; When a control node comes into one of these states (I, N, W), its offspring nodes will also become a corresponding state; When a control node is executed, its status will become $\mathrm{E}$ and one or more child nodes will be executed; When all of child nodes comes into one of these states (F, N), the control node's status will become F. (Except the loop pattern, its status will become J)

\section{The implementation algorithm of workflow model based on binary tree}

The execution of the workflow process is divided into the starting process after the instantiation and the state transitions when executing the nodes. Based on the model, two algorithms used commonly are introduced.

Node execution algorithm. The algorithm takes the node as input, which satisfies the conditions of execution. The description is as the following:

Step 1: Set the status of the current node to E.

Step 2: Identify the type of the current node: If the type is D, the algorithm ends; If the type is $\mathrm{S}$ or $\mathrm{L}$, traverse child nodes of the current node from left to right until we find the first node whose status is I or W. if there is, set the selected node as the current node and take it as input to call the algorithm recursively; If the type is $P$, traverse child nodes of the current node and find all nodes whose status is 
I or W. if there is, take each of them as input in turn to call the algorithm recursively; If the type is B, set the status of the current node to $\mathrm{J}$.

State transition algorithm. The algorithm takes the node as input, which is going to be finished. The description is as the following:

Step 1: set the status of the current node to F.

Step 2: check whether the current node has the parent node or not: If has, go to step 3; If not, which means all nodes is finished, the algorithm ends.

Step 3: Identify the type of the parent node: If the type is S, check whether the current node has the right node or not: If has, execute the right node. If not, set the parent node as the current node and take it as input to call the algorithm recursively; If the type is P, traverse brother nodes of the current node and check whether there is any node whose status is neither $\mathrm{F}$ nor N. If exist, the algorithm ends. If not, set the parent node as the current node and take it as input to call the algorithm recursively; If the type is $\mathrm{B}$, set the parent node as the current node and take it as input to call the algorithm recursively; If the type is L, check if the current node has the right node or not: if has, execute the right node. Otherwise, set the status of the parent node to $\mathrm{J}$.

\section{The Application of the Workflow Model Based on Binary Tree}

Through the above definition and modeling to workflow, we can apply them to the process management in PDM. In order to describe the application of workflow above, we use a simple project template as an example. The project consists of four phases: Quote Request, Information Collection, Quote Export and Bidding Result. And each of these phases has one or more business-related activities, such as quote request and material list. The workflow is depicted as figure 3.

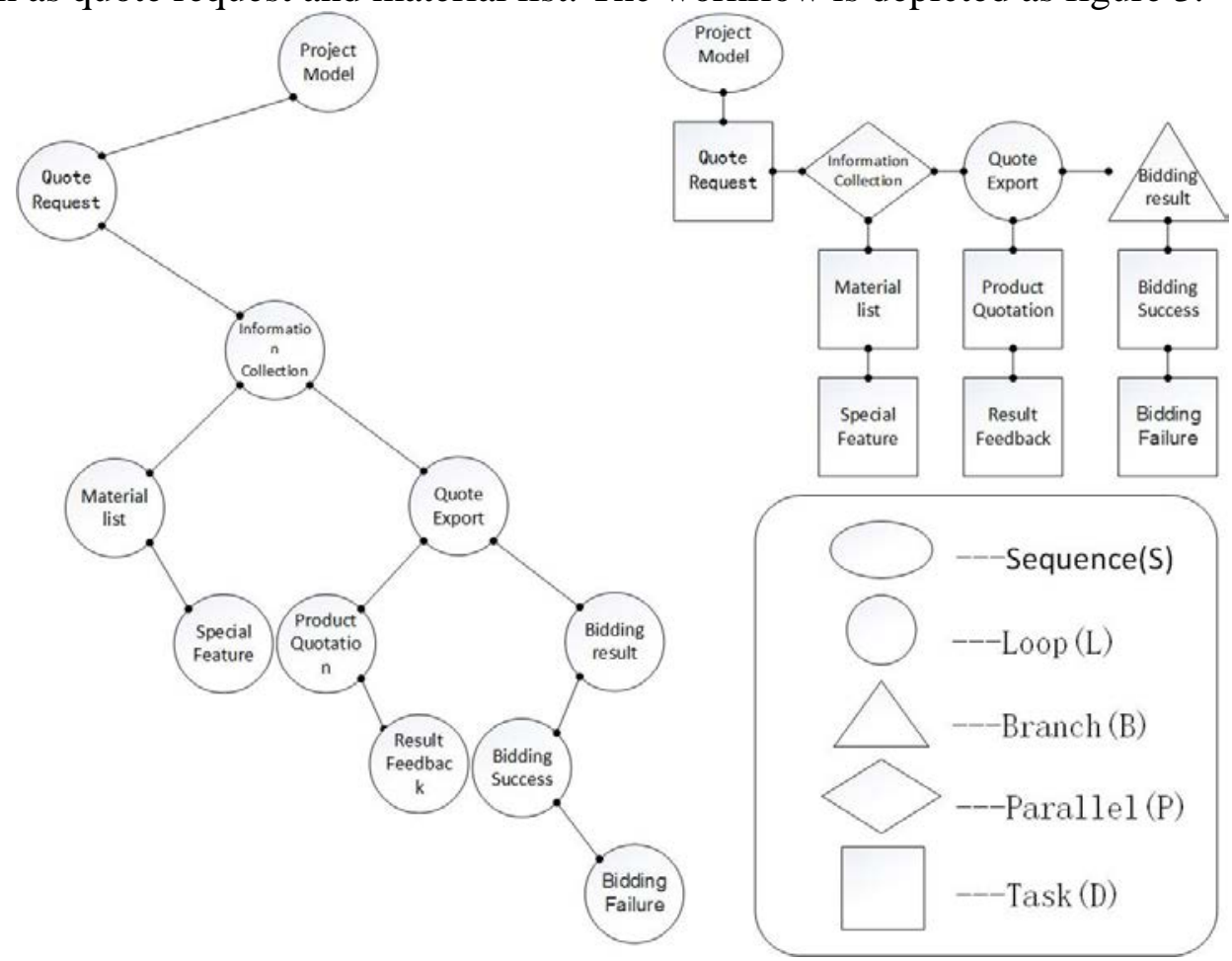

Fig. 1 Instantiated workflow

The instantiated workflow model formed binary tree. All of the nodes are in the initial state (I) at the beginning. With the execution of the workflow, states of nodes are continually changing. At last, the state of the root node changes into F, which means that the entire workflow is completed. The process can be described as follows:

(1) Start the workflow. The state of the root node changes from I into E. According to the Node Execution Algorithm in 3.1, Quote Request gets executed as a task node.

(2) When the business-related activity of Quote Request is completed, the state of the node becomes F. According to the State Transition Algorithm in 3.2, Information Collection is executed. 
Because the type of Information Collection is P, Material List and Special Feature get executed in parallel.

(3) Until both Material List and Special Features are finished, the state of Information Collection becomes $\mathrm{F}$ according to the State Transition Algorithm in 3.2. At the same time, quote export is executed. Because the type of Quote Export is L, Product Quotation and Result Feedback get executed in sequence.

(4) When Result Feedback is completed, the state of Quote Export becomes J. At this point, the node has to determine whether loop or not according to the result. If the result meets the condition, the execution gets out of the loop and continues. If not, the execution returns to the beginning of the loop and Product Quotation gets executed again.

(5) When the state of Quote Export becomes F, Bidding Result is executed according to the State Transition Algorithm in 3.2. Because the type of Bidding Result is B, the state of Bidding Result becomes J. At this point, the node has to determine to execute Bidding Success or Bidding Failure. The selected node is going to be executed and the other becomes $\mathrm{N}$.

(6) When Bidding Success or Bidding Failure is completed, the state of bidding result and the state of its parent node become F according to the State Transition Algorithm in 3.2. Because Project Model, as the parent node of Bidding Result, is the root node of the binary tree, the entire workflow is finished.

Through the process above, we can find that workflow model based on binary tree is easy to implement the overall control of process and has a good adaptability to process changes.

\section{Summary}

Workflow model based on the structure of binary tree is proposed in this paper. By combining the control structure and the structure of binary tree, implement the overall control of the process. Also, Due to the characteristics of the structure of binary tree, the model has a good adaptability to process changes. The model can be mapped to the structure of Petri nets to validate its efficiency and completeness. In the future work, we will optimize the structure and height of the tree to enhance efficiency and user privilege control will be introduced into this model to extend the scope of application.

\section{References}

[1] Eckert, C., P. J. Clarkson, and W. Zanker, 2004. "Change and Customisation in Complex Engineering Domains.” Research in Engineering Design 15(1):1-21.

[2] Fei He. Workflow Model Design based on N-tree for Process Management in PDM[C]. Advanced Materials Research Vols,2011,268-270.

[3] LIU Jun, TANG Xiao-an, GAN Zhe, LI Xiao-hui. Research of Workflow Model Based on Activity-NetWork-Based Diagram [J]. Microcomputer Information,2009,09:9-11.

[4] WANG Ying-xia. A Event-Driven Design and Implementation for Workflow Management System[J]. Computer Technology and Development.2012,02:18-21.

[5] PAN Qishu, JIANG Bing.Petri net based workflow modeling techniques and applications[J]. JOURNAL OF TSINGHUA UNIVERSITY (SCIENCE AND TECHNOLOGY),2000,09:86-89.

[6] YUAN Huan-wu,FAN Hong-li. The Application of PDM Workflow in a Corporation [J]. Techniques of Automation and Applications,2006,04:25-29. 